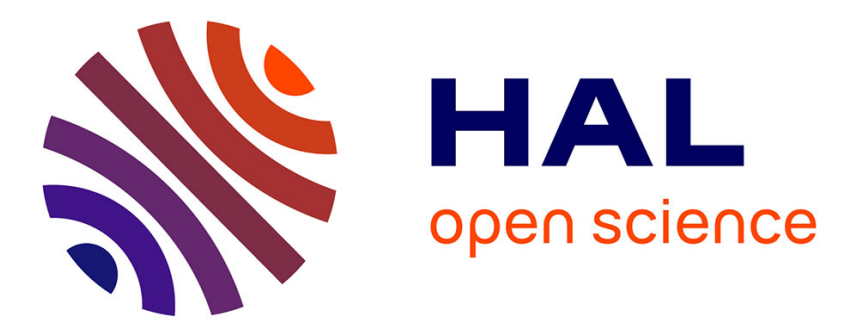

\title{
An Automated Assay for the Evaluation of Mortality in Fish Embryo
}

Élodie Puybareau, Marc Léonard, Hugues Talbot

\section{To cite this version:}

Élodie Puybareau, Marc Léonard, Hugues Talbot. An Automated Assay for the Evaluation of Mortality in Fish Embryo. ISMM 2015, May 2015, Reykjavik, Iceland. 10.1007/978-3-319-18720-4_10 . hal-01154543

\section{HAL Id: hal-01154543 \\ https://hal.science/hal-01154543}

Submitted on 22 May 2015

HAL is a multi-disciplinary open access archive for the deposit and dissemination of scientific research documents, whether they are published or not. The documents may come from teaching and research institutions in France or abroad, or from public or private research centers.
L'archive ouverte pluridisciplinaire HAL, est destinée au dépôt et à la diffusion de documents scientifiques de niveau recherche, publiés ou non, émanant des établissements d'enseignement et de recherche français ou étrangers, des laboratoires publics ou privés. 


\title{
An automated assay for the evaluation of mortality in fish embryo
}

\author{
Élodie Puybareau ${ }^{1}$, Marc Léonard $^{2}$, and Hugues Talbot ${ }^{1}$ \\ 1 Université Paris-Est / ESIEE \\ 2 Boulevard Blaise-Pascal, 93162 Noisy-le-Grand Cedex, France \\ ${ }^{2}$ L'Oréal Recherche et Développement \\ Aulnay-sous-Bois, France
}

\begin{abstract}
Waterways are often first and severely affected by pollution. In this context, fish embryos - which constitute a good model for sensitivity to chemicals - are widely used in environmental toxicology studies. Such studies are devoted to the analysis of a wide spectrum of physiological parameters, for instance mortality ratio. In this article, we develop an assay to determine the mortality rate of Medaka embryo. Based on video sequences, our purpose is to obtain reliable, repeatable results in a fully automated fashion. To reach that challenging goal, we develop an efficient morphological pipeline that analyses image sequences in a multiscale paradigm, from the global scene to the embryo, and then to its heart, finally analysing its putative motion, characterized by intensity variations. Our pipeline, based on robust morphological operators, has a low computational cost, and was experimentally assessed on a dataset consisting of 660 images, providing a success ratio higher than $99 \%$.
\end{abstract}

Keywords: Toxicology, medaka, image stabilisation, change detection, connected filtering.

\section{Introduction}

Environmental toxicology are of increasing importance in the cosmetics industry [1. It has become a mandatory requirement to warrant the innocuity of the products that they manufacture and sell [2], including the by-products of their manufacturing and their entire lifecycle, up to their eventual release in the environment [3]. Additionally, there are severe limits on the type of living organisms that can be used for such studies [4. Among those that are permitted for such usage, some species of transparent fish embryos constitute a good compromise. They do not require difficult husbandry techniques, they are readily available, belong to the vertebrata subphylum (like humans), and their living milieu makes them a good model to study the consequences of waterways pollution [5]. In addition they are of intermediate size (a few $\mathrm{mm}$ long), and are transparent, so they do not require high-power microscopes or sophisticated image acquisition devices. Their circulatory system, in particular, is readily visible. As a result, they have been used in toxicology for a number of years [6]. 
However, longitudinal studies of such embryos and vertical studies of the effects of various chemical compounds on their health and behavior require a lot of measurements, and these are only poised to increase dramatically. In the past, measurements were conducted manually or semi-automatically, but these approaches do not scale [7]. Recent progress in the automation of image analysis procedures with Medaka [5] or more commonly Zebrafish 89 is a natural consequence given the increasing demand in image-based toxicology assays. In short, the cosmetics industry is in need of effective, fast and reliable way to assess the vital signs of fish embryos; automated image analysis may provide answers to this need.

A beating heart is clearly one of the most basic vital signs. In this article, we propose the design of an image processing pipeline based on mathematical morphology [10:11]12] to assess the presence or absence of a beating heart pattern in fish embryo. We describe, investigate and propose solutions for all the problems and challenges we have encountered in this study. Another objective of this article is to showcase the design of an automated pipeline for processing sequences of fish embryo and more generally to provide some guidelines for the design of robust image analysis pipelines, through the particular example of a real-world problem.

The remainder of the article is as follows. In Section 2, we state our problem and list some of the difficulties we encountered. Section 3 lists our materials and methods. Section 4 provides an overview of our solution. Section 5 shows our results, together with validation and discussion. We conclude in Section 6

\section{Problem statement and difficulties}

In this article, we study short sequences of Medaka (Oryzia latipes) placed in 24-well plates handled by a movable platform under a low-power, fixed macro camera. The plates are illuminated from below. The objective is to find out if every embryo in the plate is alive or not. For this, we rely on the fact that alive embryo should exhibit a visibly beating heart. The uncompressed video sequences are acquired one by one by moving the platform under the fixed camera. The embryos are anesthetized to prevent swimming. Since they are transparent we expect that the heart should be visible under the vast majority of poses.

In many real-world applications, the available hardware was designed for a previous task, and replacement or upgrade is not a possibility. In our case, the platform was initially designed for single-frame acquisition and processing, and not designed to acquire, process or store large streams of video. In particular, the camera is limited to $15 \mathrm{fps}$, and there is only limited storage. As a result, we faced a real problem of memory management and timely processing due to image size. Hence, we reduced the acquisition to 2 seconds at 15 frames per second and cropped the frames to $800 \times 600$, the camera recording only the center of the field of view. The heart of medakas usually beats at around 130 beat per minute (bpm) but in extreme cases it can reach $300 \mathrm{bpm}$, i.e. $5 \mathrm{~Hz}$. The Nyquist frequency at $15 \mathrm{fps}$ is $7.5 \mathrm{~Hz}$, so the margin is sufficient but small. 
Because of these acquisition and memory restrictions, embryos need to be centered in the field to avoid unusable partial views during acquisition. Compounding this problem, even though we should expect embryos, even live ones, to remain immobile while they are anesthetized, they may still exhibit some residual motion. These can be involuntary reflex swimming (incomplete anesthesia). Artefactual motion can also be due to poorly controlled platform motion inducing sliding, vibrations, shocks due to the general environment of the lab. These may induce false positive on dead embryos. In particular, since the eyes of Medaka are not transparent, this fact can cause significant difficulties. First, while still in egg form, embryos appear tightly wound and eyes can obscure the heart, making the detection of a heartbeat impossible. It is important for the application to know in advance if a well contains an egg or an embryo. On hatched embryos, eyes constitute the darkest part of their body. As a result, their contour have high contrast. In the sequel, we will see that we rely on variance measures to detect the heartbeat. However, in case of even small or insufficiently compensated frame motion, this artificially creates areas of high variance and, in the end, generates false positives. Unfortunately, the heart is fairly close to the eyes in Medaka, so this problem needs to be handled carefully.

During experimental runs, illumination is manually set by the operator. Hence, it may vary from one experiment to the next, and so the level of electronic noise and image contrast can vary as well. Specifically, strong noise over the embryo body can induce false positives. Simply looking for grey-level variability in the heart region is not robust enough.

As in any real application, sometimes the hardware does not provide expected data. Here incomplete videos can sometimes be acquired, and a whole part of the sequence may appear black or the sequence may be short.

\section{$3 \quad$ Material and Methods}

\subsection{Material}

Organism Medaka (Oryzia latipes) is a small fish member of genus Oryzias. In adulthood, it can reach $4 \mathrm{~cm}$ in length, and live in both oceans and rivers. It develops quickly (7 days). In our study, only embryos coming from Amagen (UMS 3504 CNRS / UMS 1374 INRA) were used. They were placed as eggs in a neutral environment with Trypan blue to detect dead eggs, and studied until 4 days after hatching. Embryos do not feed, so no nutrients were supplied. They do excrete, so they were placed and manually centered in a new, clear well on a thin gelified bed to prevent sliding shortly before analysis.

Computer and software We used the Python 2.7.6 environnement under Windows XP in a HP computer with a Core i7 920 CPU and 4GB of RAM. We used the Numpy, Scipy, Scikit-image 13, Pink [14, and OpenCV for Python. Platform control and image data acquisition was performed using FEI Visilog 7 . Inter-process communication was handled through the python COM+ interface via Microsoft Excel. 
Hardware All videos were acquired using a color camera LEICA DFC 300 FX and an acquisition platform purpose-built by FEI .

\subsection{Protocol of acquisition}

Fish embryos were anesthetized with tricaïne $(0.1 \mathrm{~g} / \mathrm{L})$ and placed on a support gel in a 24-well plate, one embryo per well. The plate was then placed under the connector board and the acquisition was automatically performed by Visilog. We recorded $2 \mathrm{~s}$ of uncompressed video sequence at 15 frame per second and a resolution of $800 \times 600$ pixels. Platform motion, still images and video sequence acquisition takes 10 s per well.

\section{Solution overview}

Because of storage constraints, one aim was to achieve the complete sequence analysis online within the same time frame as the acquisition, i.e. in under 10s. We therefore propose a robust pipeline suitable for production usage. It consists of simple operators, which are for the most part readily available and fast. Figure 1 presents the flowchart of our assay. It is split into two phases: a preprocessing step which consists of sequence stabilization and denoising, while the actual processing step consists of detecting significant periodic changes in the embryo (variation of grey level values) assuming they are caused by its beating heart. In this section, we will use standard notations. Let $I, J$ be grey level images defined on a support $\Omega$, taking 8-bit discrete values, i.e. $I, J: \Omega \rightarrow \mathbb{Z} \cap[0,255]$. $\delta_{\mathcal{B}}(I)$ is the dilation of $I$ by the structuring element $\mathcal{B}, \epsilon_{\mathcal{B}}(I)$ is the adjunct erosion, $\gamma_{\mathcal{B}}(I)$ and $\varphi_{\mathcal{B}}(I)$ the corresponding morphological opening and closing [15], $\gamma^{r}(I, J)$ is the geodesic reconstruction by dilation of $I$ under $J$ (which is an algebraic opening). $\gamma^{\lambda}(X)$ is the area opening of $X$ with parameter $\lambda$ [16]. We also introduce the Boolean image $I_{\geq \theta}=\left\{\forall x \in \Omega, I_{\geq \theta}(x)=(I(x) \geq \theta)\right\}$, i.e. the thresholded image of $I$ at value $\theta$. To implement the connected operators [17]18, an efficient max-tree/min-tree framework is used [19].

\subsection{Segmentation of the embryo}

The first step is an initial segmentation of the embryo. Indeed, this segmentation is crucial for several reasons. In order to reduce the memory usage, we need to crop the area of interest to a small window centered on the embryo. This step allows us to weed out the sequences where an embryo is not visible or intersects the acquisition window. Moreover, we generally need to stabilize the sequence, and this stabilization must be performed on the embryo itself, and not other elements on the field of view like the contours of the well. The background appears white whereas edges of the well and embryo are darker (see Fig. 2(a)), particularly the eyes are very dark. Therefore the embryo is easy to segment as the largest connected component in the min-tree associated with the darkest minimum not connected to the edges of the field of view, simultaneously maximizing 


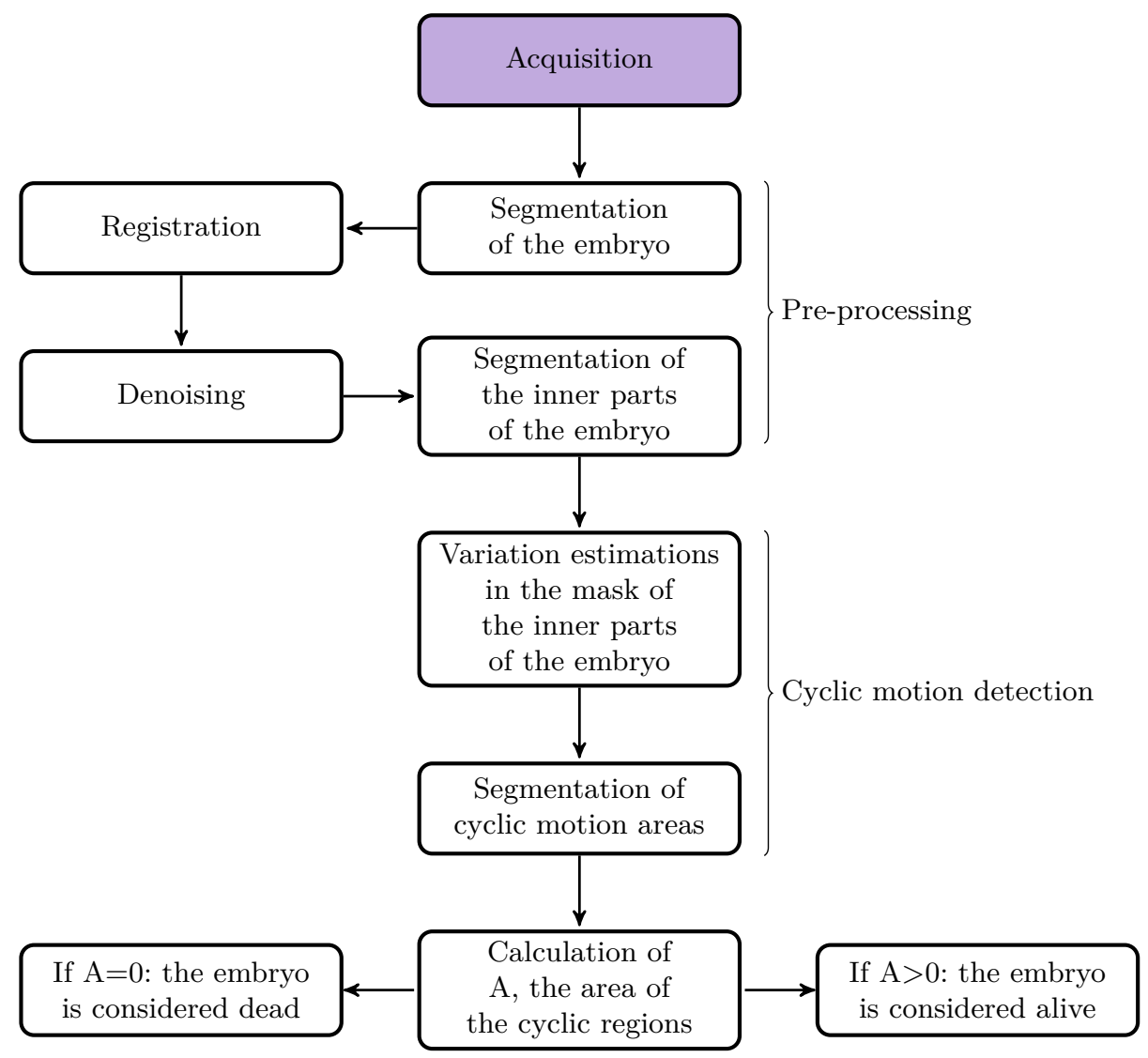

Fig. 1. Flowchart of our embryo mortality image processing assay.

the inter-class variance between foreground and background (i.e, following the Otsu criterion [20]). We implemented it as follows. If $\theta_{O}$ is the Otsu threshold for the first input frame $S^{0}$, then

$$
A^{0}=\mathbb{1}-S_{\geq\left(\theta_{O}+\theta_{b}\right)}^{0},
$$

where $\mathbb{1}$ is a boolean image consisting only of ones, and $\theta_{b}$ is a baseline value, optimized to 30 experimentally.

Because we are not interested in the thin elements of the embryo (i.e. the tail), a morphological opening is applied, and because a single connected component is desired, an area criterion is also used:

$$
A^{1}=\varphi_{\mathcal{B}_{1}}\left(\varphi_{\lambda_{1}}\left(A^{0}\right)\right) \text {. }
$$

Experimentally, we determined the average area of an healthy embryo to 3000 pixel, so we set $\lambda_{1}$ conservatively to 600 pixel. The embryo's body is between 
20-60 pixel wide, while artifacts in the well are usually much smaller, so $\mathcal{B}_{1}$ is set to a discrete Euclidean ball of radius 3. Since the embryo is expected to be near the center of the field of view, we remove all objects connected to the frame of the image, calling the result $A^{2}$. For speed, all these operators are implemented on the min-tree structure of the image, except the opening with $\mathcal{B}_{1}$, which is approximated by a fast, separable dodecagon [21.

This result $A^{2}$ is expected to represent the mask of the embryo (see Fig 2 (b)). However, if the result is empty, this means that the embryo is not centered in the well and then the sequence cannot be reliably analyzed. If we do find an embryo, we crop the sequence by defining a bounding box around our segmentation, dilated by 10 pixels. The result is a new sequence $S^{1}$ centered on the thorax of the embryo (see Fig 2(c)).

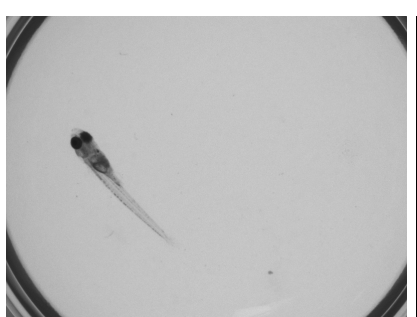

(a) Initial frame.

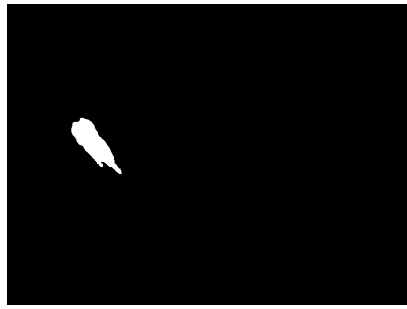

(b) Embryo segmentation.

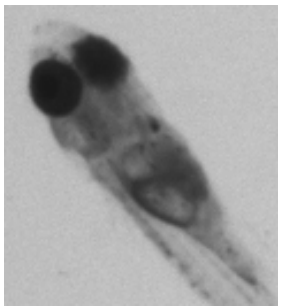

(c) Cropped frame.

Fig. 2. Segmentation of the initial frame to locate and the embryo in the well.

\subsection{Registration}

Because of vibrations associated with other equipment around the acquisition platform, the embryo can appear to slide up and down in its well. The sequence needs to be stabilized to avoid false positives. For speed, availability and ease of use, we have chosen the SIFT key-point based method [22. This method detects remarkable points on each frame and matches sets of pairs of points $P_{1}$ and $P_{2}$ between two frames. We then solve the rigid transformation equation:

$$
P_{1}=P_{2} \times \mathcal{R}+\mathcal{T}
$$

Where $\mathcal{T}=\left(d_{x}, d_{y}\right)$ is the translation and $\mathcal{R}$ the rotation matrix. We consider only rigid transformations because motion consists only of vibrations and the embryo does not deform. Our model allows to select between both translationrotations and translation only. If rotation appears negligible (i.e. the sum of square differences between the registration using translation-rotation and translation-only models are not significantly different), then only the translation $\mathcal{T}$ between $P_{1}$ and $P_{2}$ is calculated while $\mathcal{R}$ is set to the identity. In our experiments, this is the case most of the time. 
We then apply the estimated transform between the first cropped frame of the sequence $S^{1}$ and all subsequent frames, taking the first as reference. We compute $d_{x \max }=\max \left|d_{x}\right|$ and $d_{y \max }=\max \left|d_{y}\right|$ for all frames, and we crop the bounding box of the whole sequence further by these amounts in $x$ and $y$ respectively. We call $\mathcal{S}^{2}$ the stabilized, cropped sequence of the embryo.

\subsection{Denoising}

To eliminate global illumination variation during a sequence, which occurs sometimes, we equalize the average intensity of all frames. We obtain a sequence $\mathcal{S}^{3}$ where the average value of all frames is constant throughout the sequence.

Depending on the average illumination, the sequence can be quite noisy, so we used a $2 \mathrm{D}+\mathrm{t}$ bilateral filter [23] to remove the noise remaining on $\mathcal{S}^{3}$.

The bilateral filter is a spatially variant convolution, where for each pixel at coordinate $(i, j)$, in a window $W$, we have:

$$
\mathcal{I}_{D}(i, j, t)=\frac{1}{\sum_{(k, l, m) \in W} w(i, j, t, k, l, m)} \sum_{(k, l, m) \in W} I(k, l, m) * w(i, j, t, k, l, m),
$$

where

$$
w(i, j, t, k, l, m)=\exp \left(-\frac{(i-k)^{2}+(j-l)^{2}+(t-m)^{2}}{2 \sigma_{d}^{2}}-\frac{\|I(i, j, t)-I(k, l, m)\|^{2}}{2 \sigma_{r}^{2}}\right) .
$$

Here $I$ is the original input image to be filtered; $\mathcal{I}_{D}$ is the denoised intensity of pixel $(i, j)$. It is crucial not to filter too much, otherwise the heartbeat might be edited out. We have optimized the parameters that removed the noise without removing the heartbeat. These are: window size $=5 \times 5 \times 5, \sigma_{r}=0.75, \sigma_{d}=0.9$. The result is a denoised sequence $\mathcal{S}^{4}$. Due to border effects, we remove the first three and the last three frames from this sequence, which is then 24-frames long.

\subsection{Segmentation of the inner parts of the embryo}

If the embryo is alive, the heart should be detected in its thorax region. To ascertain this, cyclic motion should be detected in this region of the sequence, but not anywhere else where remaining noise or motion might be present. Because eyes are very dark, noise causes detectable variations in them, and remaining motion may be detectable near the contours of the embryo. We developed a mask $\mathcal{M}^{1}$ of the inner part of the embryo to avoid false detection in these areas.

We define $B^{1}$ as the minimum image of the sequence: $B^{1}=\min \left(\mathcal{S}^{4}\right)$. In this image, where the heart and vessels are darker because of the presence of blood, we apply the same procedure as in section 4.1 i.e:

$$
B^{2}=\varphi_{\mathcal{B}_{1}}\left(\varphi_{\lambda_{1}}\left(\mathbb{1}-B_{\geq\left(\theta_{O}+\theta_{c}\right)}^{1}\right)\right)
$$

In this instance, because of the change of contrast, $\theta_{c}$ is set to $20 ; \theta_{O}$ is again obtained using the Otsu criterion; $\lambda_{1}$ and $\mathcal{B}$ are unchanged. The resulting $B^{2}$ 
is a binary mask (with values in $\{0,1\}$ ) of the registered body of the embryo. Considering $B^{2}$ as a geodesic mask, we now segment the eyes as the one or two more prominent minima from the min-tree. We cannot rely on the eyes being separated. Depending on the pose of the embryo, they might get merged. We write:

$$
\mathcal{M}^{1}=\epsilon_{\mathcal{B}_{3}}\left(\gamma_{\mathcal{B}_{2}}\left(\left(B^{2} \cdot B^{1}\right)_{\geq\left(\theta_{O}-\theta_{c}\right)}\right)\right)
$$

Here the dot in $B^{2} \cdot B^{1}$ denotes the pixelwise multiplication. Note that $\theta_{O}$ is re-estimated from the grey-level distribution of the embryo within the geodesic mask. In this formula, $\mathcal{B}_{2}$ is a ball of radius 1 and $\mathcal{B}_{3}$ a ball of radius 3 . The outline of the resulting mask $\mathcal{M}^{1}$ are exemplified on Fig. 3 .

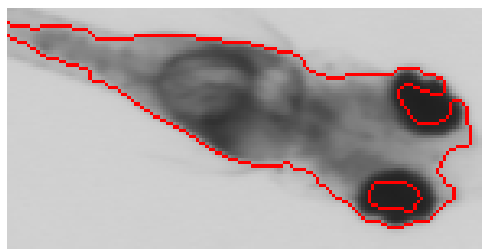

(a)

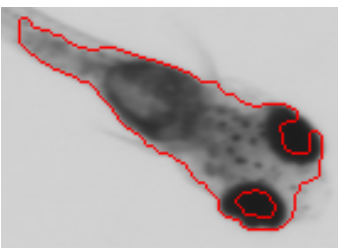

(b)

Fig. 3. Inner parts segmentation on two embryos. (a) is alive whereas (b) is dead.

\subsection{Variation estimations in the mask of the inner parts of the embryo}

Our challenge is now to detect cyclic motion-induced variations in the sequence assuming it corresponds to a heartbeat. The heartbeat is noticeable by changes of contrast due to blood cell concentration in the heart region. Computing the grey level variance at each pixel of a sequence along the time line show the greylevel variability for that pixel, however this variability may not be due to a cyclic pattern. To account for cyclic variations, we split $\mathcal{S}^{4}$ into 3 sub-sequences of 8 frames $\left(\mathcal{S}_{1}^{4}, \mathcal{S}_{2}^{4}\right.$ and $\left.\mathcal{S}_{3}^{4}\right)$. An interval of 8 frames is comparable to the expected period of a single heart beat. We compute the pixels variance inside $\left(\mathcal{M}^{1}\right)$ on each sub-sequence, yielding three variance images $\mathcal{V}_{1}, \mathcal{V}_{2}, \mathcal{V}_{3}$. We next compute the median of these three images $\mathcal{V}_{i}$. In this way, some spurious, potentially even large, residual variation occurring only once in the sequence will produce a large variance only once, and so the median of all the variances in this area will remain small. On the other hand, cyclic variation will exhibit significant variance in all 
three sub-sequences.

$$
\begin{aligned}
\mathcal{S}^{4} & =\left\{\mathcal{S}_{1}^{4} \cup \mathcal{S}_{2}^{4} \cup \mathcal{S}_{3}^{4}\right\} \\
\forall i \in[1,3], \mathcal{V}_{i} & =\operatorname{variance}\left(\mathcal{S}_{i}^{4}\right) \\
C^{1} & =\mathcal{M}^{1} \cdot \operatorname{median}\left(\mathcal{V}_{i}, i \in[1,3]\right)
\end{aligned}
$$

The result of this process is shown in Fig. 4

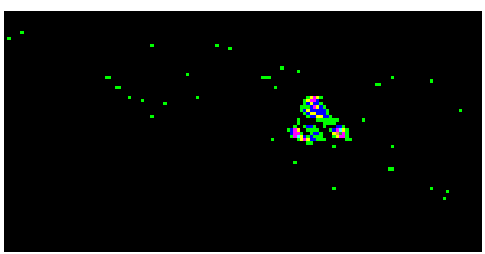

(a)

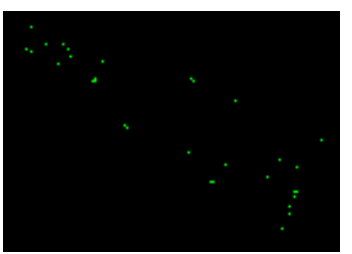

(b)

Fig. 4. False color rendering of the temporal variance. (a) is for a living embryo, (b) a dead one.

\subsection{Segmentation of cyclic motion areas}

Binarizing the image of the cyclic variance is simple with area openings and a small closing in classical alternating sequence.

$$
D^{1}=\left(\gamma^{\lambda_{3}}\left(\varphi_{\mathcal{B}_{4}}\left(\gamma^{\lambda_{2}}\left(C^{1}\right)\right)\right)\right)_{\geq 1} .
$$

Here, $\lambda_{2}=3, \mathcal{B}_{4}$ is a radius- 2 ball, and $\lambda_{3}=10$. This image is then thresholded at 1 .

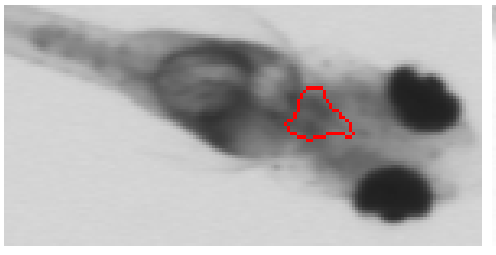

(a)

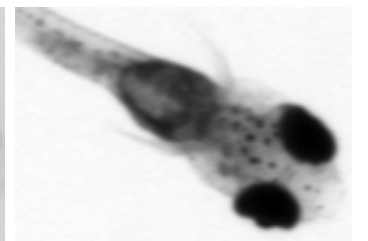

(b)

Fig. 5. Segmentation of cyclic motion detection on embryos. (a) corresponds to the segmentation of 4 (a) (living embryo), and (b) corresponds to the segmentation of 4 (b) (dead embryo)

If the number of non-zero pixels in $D^{1}$ is zero, we consider the embryo is dead, otherwise, it is considered alive. 


\subsection{Parameter optimization}

In our pipeline, the parameters such as the size of the structuring elements or thresholds need to be optimized. Many of them are dependent on the size of the embryo. For the remaining parameters, they were hand-optimized on a training sample of 100 sequences.

\section{Results and Validations}

\subsection{Sequence extraction}

Videos are read as raw data interpreted as grey-level values. If the maximum intensity of any frame is zero, the video is deemed incomplete and discarded.

\subsection{Processing}

We tested our program on 651 videos taken over several experimental runs. The protocol was identical each time but conditions such as illumination or marker concentration could change. This number of videos is not insignificant and reflect production usage. We have also tested our protocol on unusual embryos: some with oedemas and with other malformations to ensure the robustness of our protocol (see Fig. 6(a) and (b)). The program processes a sequence in less than 10 seconds, which is in accordance with our initial constraints.

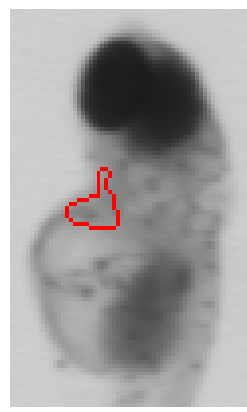

(a)

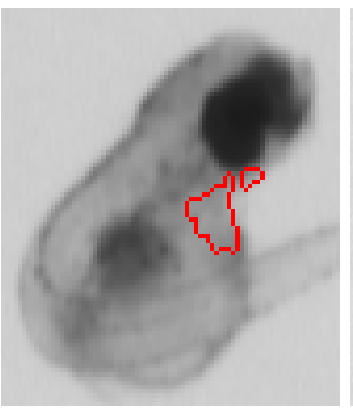

(b)

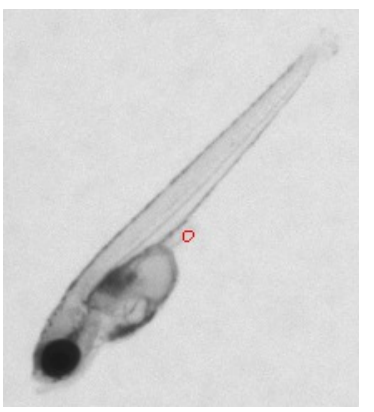

(c)

Fig. 6. Heart segmentation in the presence of oedema (a) or malformation (b). Incorrect segmentation in the fin due to fluttering (c).

\subsection{Limitations}

In some cases, even actually dead embryos can appear to move. This can be due to the gel or the presence of shadows (figure 6.(c)). In dark areas, electronic noise is more prevalent, and may appear as motion and in some cases, the embryo may appear to slide on the gel. This happens if we incorrectly do not compensate 
for rotation in the sequence stabilization phase. The main remaining cause of problem is ambiguity: in some cases, the heart beats so slowly or weakly that we cannot detect it. In most cases, a human operator would also have difficulties in detecting it. We could also argue that embryo with weak heartbeats are not very healthy and likely to die, however this is unconfirmed.

\subsection{Validation of the method}

We have run experiments on many sequences: some darker, some lighter, some with varying amounts of tricaine, some with embryo abnormalities, and so on. In total, we processed 651 such sequences, which might be considered sufficient for a first validation step.

From an initial set of 660 videos, 9 were eliminated as incomplete. From the remaining set of 651 sequences, 100 were used for training, i.e. optimizing the parameters of the pipeline. The remaining 551 were used for testing. There was 1 remaining error in the training sample ( $1 \%$ error rate). There were 3 errors in total in the test set, for an error rate of $0.54 \%$. Such an error rate is effectively quite low.

\section{Conclusion}

In this article, we developed a solution for the fish embryo version of Schrödinger's problem. In effect we have proposed a simple and effective image analysis pipeline to detect whether a Medaka embryo is alive or not, by detecting its heart region and finding if cyclic variations were present there. The proposed procedure was optimized on 100 sequences and tested on more than 550. The error rate compared with careful manual checking is near $0.5 \%$, which is sufficient for production use. Based on this result, a more capable sequence acquisition platform is currently being built at L'Oreal, with an acquisition capacity of thousands of sequences per week, for use in toxicology studies. As future work, we believe that with improved acquisitions, the heart rate would also be measurable.

\section{References}

1. Indans, I.: The use and interpretation of in vitro data in regulatory toxicology: Cosmetics, toiletries and household products. Toxicology Letters 127(1-3) (2002) 177-182

2. Buzek, J., Ask, B.: Regulation (ec) no 1223/2009 of the European Parliament and of the Council of 30 november 2009 on cosmetic products. Official Journal of the European Union L 342 (2009)

3. Ramirez, T., Kolle, S.N., Schulte, S., van Ravenzwaay, B., Schulz, M., Mehling, A., Kleber, M., Teubner, W., Landsiedel, R.: Toxicological testing of cosmetic ingredients using alternative methods - The perspective of a supplier. IFSCC Magazine 16(4) (2013) 263-274 
4. Lilienblum, W., Dekant, W., Foth, H., Gebel, T., Hengstler, J.G., Kahl, R., Kramer, P.J., Schweinfurth, H., Wollin, K.M.: Alternative methods to safety studies in experimental animals: Role in the risk assessment of chemicals under the new European Chemicals Legislation (REACH). Archives of Toxicology 82(4) (2008) $211-236$

5. Oxendine, S.L., Cowden, J., Hinton, D.E., Padilla, S.: Adapting the medaka embryo assay to a high-throughput approach for developmental toxicity testing. NeuroToxicology 27(5) (2006) 840-845

6. Dial, N.A.: Methylmercury: Some effects on embryogenesis in the Japanese Medaka,Oryzias latipes. Teratology 17(1) (1978) 83-91

7. Schindelin, J., Arganda-Carreras, I., Frise, E., Kaynig, V., Longair, M., Pietzsch, T., Preibisch, S., Rueden, C., Saalfeld, S., Schmid, B., Tinevez, J.Y., White, D.J., Hartenstein, V., Eliceiri, K., Tomancak, P., Cardona, A.: Fiji: An open-source platform for biological-image analysis. Nature Methods 9(7) (2012) 676-682

8. Liu, T., Lu, J., Wang, Y., Campbell, W.A., Huang, L., Zhu, J., Xia, W., Wong, S.T.: Computerized image analysis for quantitative neuronal phenotyping in zebrafish. Journal of Neuroscience Methods 153(2) (2006) 190-202

9. Xia, S., Zhu, Y., Xu, X., Xia, W.: Computational techniques in zebrafish image processing and analysis. Journal of Neuroscience Methods 213(1) (2013) 6-13

10. Serra, J.: Image analysis and mathematical morphology. Academic Press (1982)

11. Soille, P.: Morphological Image Analysis, principles and applications. Springer (1999)

12. Najman, L., Talbot, H., eds.: Mathematical Morphology: From theory to applications. ISTE-Wiley, London, UK (September 2010) ISBN 978-1848212152.

13. Van der Walt, S., Schönberger, J.L., Nunez-Iglesias, J., Boulogne, F., Warner, J.D., Yager, N., Gouillart, E., Yu, T., the scikit-image contributors: Scikit-image: Image processing in Python. PeerJ 2 (6 2014) e453

14. Couprie, M., Marak, L., Talbot, H.: The Pink Image Processing Library. In: Euroscipy, Paris (2011)

15. Heijmans, H.: Morphological image operators. Advances in Electronics and Electron Physics Series. Academic Press, Boston (1994)

16. Vincent, L.: Grayscale area openings and closings, their efficient implementation and applications. In: Proceedings of the conference on mathematical morphology and its applications to signal processing, Barcelona, Spain (1993) 22-27

17. Salembier, P., Oliveras, A., Garrido, L.: Antiextensive connected operators for image and sequence processing. IEEE Transactions on Image Processing $7(4)$ (1998) 555-570

18. Jones, R.: Connected filtering and segmentation using component trees. Computer Vision and Image Understanding 75(3) (1999) 215-228

19. Najman, L., Couprie, M.: Building the component tree in quasi-linear time. IEEE Transactions on Image Processing 15(11) (2006) 3531-3539

20. Otsu, N.: A threshold selection method from gray-level histograms. Automatica 11(285-296) (1975) 23-27

21. Jones, R., Soille, P.: Periodic lines: Definition, cascades, and application to granulometries. Pattern Recognition Letters 17(10) (1996) 1057-1063

22. Lowe, D.G.: Object recognition from local scale-invariant features. In: IEE ICCV. Volume 2. (1999) 1150-1157

23. Tomasi, C., Manduchi, R.: Bilateral filtering for gray and color images. In: Computer Vision, 1998. Sixth International Conference on, IEEE (1998) 839-846 